\title{
Towards a complementary balanced energy harvesting solution for low power embedded systems
}

\author{
Yannick Verbelen • An Braeken • Abdellah Touhafi
}

Received: 1 August 2013 / Accepted: 24 January 2014 / Published online: 19 February 2014

(C) The Author(s) 2014. This article is published with open access at Springerlink.com

\begin{abstract}
The specific technical challenges associated with the design of an ambient energy powered electronic system currently requires thorough knowledge of the environment of deployment, energy harvester characteristics and power path management. In this work, a novel flexible model for ambient energy harvesters is presented that allows decoupling of the harvester's physical principles and electrical behavior using a three dimensional function. The model can be adapted to all existing harvesters, resulting in a design methodology for generic ambient energy powered systems using the presented model. We also present a solution for the mathematical problem involved with the optimization of generator sizes when more than two harvesters are used, and demonstrate the ease of use of this solution for implementations on embedded systems with few system resources. Concrete examples are included to demonstrate the versatility of the presented design in the development of electronic appliances on system level.
\end{abstract}

\section{Introduction}

Embedded systems with low power requirements are currently often equipped with a finite energy source, practically almost always one or more chemical cells acting as the only power source for the system. Aside from well

Y. Verbelen $(\bowtie) \cdot A$. Braeken · A. Touhafi

Vrije Universiteit Brussel, Pleinlaan 2,

1050 Etterbeek, Brussels, Belgium

e-mail: yannick.verbelen@vub.ac.be

A. Braeken

e-mail: an.braeken@vub.ac.be

A. Touhafi

e-mail: abdellah.touhafi@vub.ac.be known environmental drawbacks, equipping embedded systems with batteries is a very expensive approach of providing them with energy, since these batteries will inevitably be depleted-either through useful work or self discharge and leakage. Especially the need for human intervention to replace depleted batteries with new ones exceeds the cost of these batteries in commercial applications (Barosso and Roedig 2004). While ongoing research is being conducted to extend the number of charge/discharge cycles of rechargeable batteries, current generation rechargeable NiMH or Lithium batteries will need replacement after 1,000 cycles (Verbelen and Touhafi 2013). For a system with a period of one day, this would imply imminent replacement is necessary within less than 3 years. There are also many ethical, environmental and economical concerns associated with the use of batteries (Verbelen et al. 2013). The increasing popularity of autonomous embedded systems, which need to have an ideally infinite lifetime (and thus power source) encourages designers to stray off the path of battery powered only systems and look beyond chemical energy sources to power their systems. Harvesting energy from the system's environment presents a viable solution for the problem of how to provide power to a system for prolonged periods of time in a reliable and economically feasible way.

In this paper, Sect. 2 starts with the identification of the problems resulting from current energy harvesting solutions, and an overview of previous work. Sections 3 and 4 suggest a solution by breaking up the behavior of harvesting generators in 3 parameters, and applying them to a system which is able to harvest energy from more than one source. Section 5 elaborates the practical aspects of multi sourced periodic systems. Section 6 discusses the problem of generator balancing and the introduction of the harvester coefficient, while Sect. 7 focuses on practical generator 
sizes. Sections 8 and 9 suggest opportunities for future work by analyzing the parametrization of generator efficiency. Finally, Sect. 10 concludes the paper with a summary of the presented work.

\section{Current energy harvesting approach}

Despite the rising awareness of developers about the opportunities provided by energy harvesting to prolong the autonomy of their applications, too often energy harvesting is simply seen as a replacement for batteries. This is evidenced by the numerous integrated energy harvesting capable modules currently existing on the market, such as those commercially distributed by EnOcean (Isaac and Mike 2011) and PowerCast (Ostaffe 2009). Many system engineers seek offthe-shelf energy harvesting solutions. Recently, plug\&play energy harvesting solutions have drawn significant attention from the industry (Clarke 2010; Tansel 2011) and energy harvesting generators having the form factor of AA or AAA batteries are often seen as ideal situations.

To maximize the power harvested from them, however, ambient energy sources demand designers to think beyond the conventional battery-electronics concept. Rather than seeing the ambient energy powered generator (hereafter addressed simply as generator or harvester) as a replacement for batteries, it is a necessity to adapt the application to the environment it is designed to operate in, and engineer the electronics with the generator as foundation. Consequently, sales of these off the shelf energy harvesting solutions have in most cases shown sales disproportional to the enthusiasm with which they were received. Ironically, on the other side of the spectrum stand alone harvesters have seen a greater success despite their lack of integration (Fowler and Sherr 2012).

Insight in the matter reveals that the highly volatile nature of ambient energy sources, combined with the requirement to tune harvesters to these sources, prevents the construction of energy harvesting black boxes. Electronics developers also often lack knowledge of the physical principles on which energy harvesters are based, further obstructing the integration of ambient energy harvesters in electronic appliances. In Sect. 3 a methodology is proposed to solve this problem for efficient use of ambient energy harvesting techniques. The scientific principles of operation are disconnected from their technological properties in order to make the application of energy harvesting more transparent.

\section{Modeling generator parameters}

Consider any generator $g$ that is able to capture ambient energy from its environment and convert it into electrical energy. The power output of $g$ is $P_{g}$, equal to the voltage over $g$ multiplied by the current that it is able to deliver at that voltage. Since the amount of ambient energy $\phi$ tends to vary, the generator's power $P_{g}(t)$ is also a function of the time. The function $P_{g}(t)$ makes it considerably more difficult to engage in the dimensioning of a system powered by $g$ since the boundaries of $P_{g}(t)$ (its minimum and maximum value) are unknown. To overcome this issue, it is necessary to break $P_{g}$ apart in a set of parameters, and then model these parameters individually to build up a practically usable model of $P_{g}(t)$.

When comparing datasheets of generators of different types, it immediately becomes apparent that the number of parameters that influences $P_{g}$ is very large: the size of the generator, its type, the amount of energy in its range, the temperature and humidity it is operating in, the efficiency with which it is able to convert ambient energy into electricity, the position of the generator with respect to the ambient energy source etc. Needless to say, practical calculations of ambient energy powered embedded systems will become increasingly complicated when hogging all these parameters into the equations, which does not help the simplification of the problem forward.

The approach presented in this paper attempts to model $P_{g}(t)$ as a function of 3 parameters, each grouping the influences of a specific nature together. We distinguish technology, environmental and application specific parameters.

\subsection{Technology specific parameters}

A collection of technology specific parameters such as the type of materials used in the construction, the quality of the materials, the scientific principles on which the generator is based, etc. are not subject to change by the application designer using the generator. These parameters are decided by the generator's manufacturer or limited by physical barriers, and determine the maximum amount of power $p_{g}$ a generator $g$ is able to capture in the most optimal conditions. The variable $p_{g}$ is a property of the generator that must be specified by the manufacturer, or can be tested by measuring the power output of a generator in reference conditions.

Within the scope of the design process of a single application, $p_{g}$ will be constant. However, as technology progresses and advances are made in the manufacturing processes of generators, $p_{g}$ gradually increases. An important practical consequence of this is that it is useless to create a "final" list of $p_{g}$ values per ambient energy type.

The value of $p_{g}$ as parameter is only useful when it is normalized because within a group of generators for the same type of ambient energy, the size of the generator can vary between manufacturers. This results in a generator power $p_{g}$ corresponding to the specific maximum power per 
unit of size, e.g. per $\mathrm{cm}^{3}$ for volume oriented generators or per $\mathrm{cm}^{2}$ for surface oriented generators.

\subsection{Environment specific parameters}

The environment specific parameters unite all external influences on the generator, such as changing atmospheric conditions and fluctuations in the ambient energy $\phi$. Section 8 further elaborates on these parameters, determining the efficiency of the generator $\eta_{g}$. As will be demonstrated, $\eta_{g}$ strongly varies in time, and is responsible for the reliability issues often associated with ambient energy powered systems.

\subsection{Application specific parameters}

The designer of an ambient energy powered electronic system cannot influence technology specific parameters defined by $p_{g}$, nor influence environment specific parameters referred to with $\eta_{g}$. There is however one parameter the designer is able to modify, specifically for the application itself, being the size of the chosen generator $\mu_{g}$.

It is important to note that a correlation between $p_{g}$ and $\mu_{g}$ exists because the performance of a generator design depends on its size. Since it is technologically easier to make power conversion mechanisms more efficient on a larger scale, the specific generator power will be a function of its total size denoted by $p_{g}\left(\mu_{g}\right)$.

\subsection{Power of an energy harvesting generator}

Let $\zeta$ be the size reference for generators, defined as $1 \mathrm{~cm}^{2}$ for surface oriented generators (such as solar panels) and $1 \mathrm{~cm}^{3}$ for volumetric generators (such as micro turbines). Since $\left[p_{g}\right]=\frac{W}{\varsigma},\left[\mu_{g}\right]=\varsigma$ and $\eta_{g}$ is a dimensionless efficiency, these parameters express the power of the generator as shown in Eq. 1 with $\left[P_{g}\right]=W$.

$P_{g}(t)=\mu_{g} p_{g}\left(\mu_{g}\right) \eta_{g}(t)$.

In this equation, $p_{g}$ denotes the normalized maximum generator power per unit of size, $\mu_{g}$ denotes the size of the generator, and hence $p_{g} \mu_{g}$ corresponds to the total maximum power a generator of size $\mu_{g}$ can produce. Figure 1 shows an example of this mechanism for the case specific power output of a fixed size photovoltaic generator in natural lighting conditions. The maximum output is reached when the generator's efficiency $\eta_{g}$ equals 1 . This expression can now be used to investigate the limitations of a single generator powered system. Note that the notation of $\mu_{g}$, using $\zeta$, is a simplification with the sole purpose of formulating a single equation to incorporate both surface oriented and volumetric generators.

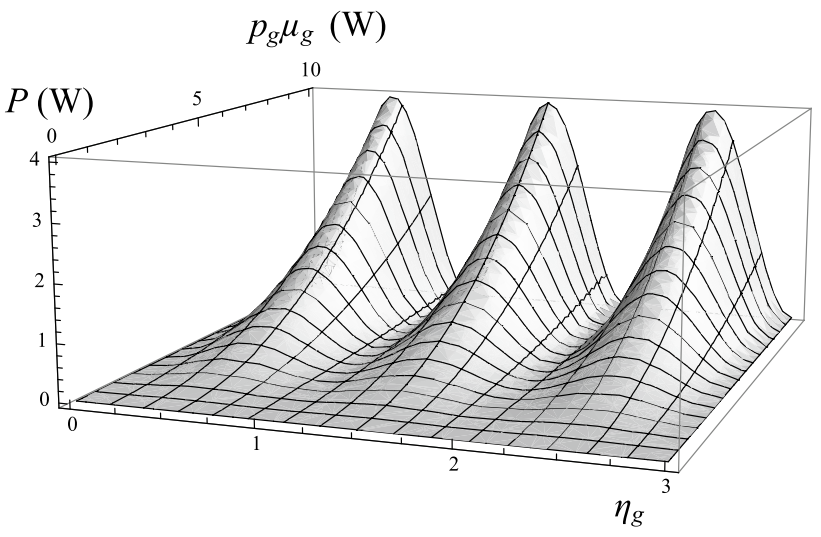

Fig. 1 Yield of a photovoltaic solar cell as a function of the efficiency $\mu_{g}$ which varies over a 3 day time interval. The applicate denotes the output power $P$ for any generator size $\mu_{g}$ assuming the maximum power output $p_{g}$ is constant $\left(\mu_{g} p_{g} \in \Re^{+}\right)$

\subsection{Example}

Assume in a subway station, it is desired to count the number of passengers that enter through a certain entrance to obtain an overview of rush hours in that specific station. After benchmarking, it is determined that the most prominently available ambient energy source is kinetic energy from the moving passengers (O'Donell 2008; Shenck and Paradiso 2001). A comparison is made between 3 harvesters for kinetic energy, denoted as generators $g_{1}, g_{2}$ and $g_{3}$.

Harvester $g_{1}$ has a maximum rating of $200 \mathrm{~mW}$ and a nominal maximum output of $182.5 \mathrm{~mW} \pm 10 \%$ and a rated output voltage of $150 \mathrm{VAC}$ for a volume of $0.821 \mathrm{dm}^{3}$ (Arveni 2013). This allows the power $p_{1}$ of this harvester to be calculated, assuming linear extrapolation, to $p_{1}=222.3$ $\mathrm{W} / \mathrm{m}^{3} \pm 10 \%$. A second harvester has a rated energy output of $2.1 \mathrm{~mJ}$ per pulse for a force of $3.4 \mathrm{~N}$ in a volume of $15 \mathrm{~mm}$ x $62 \mathrm{~mm} \times 10 \mathrm{~mm}$ (Arveni 2012). With a pulse with of $250 \mathrm{~ms}$ (Arveni 2012) this results in a yield of $8.4 \mathrm{~mW}$ for a volume of $9.3 \mathrm{~cm}^{3}$ which is equivalent to a power $p_{2}$ $=91 \times 10^{2} \mathrm{~W} / \mathrm{m}^{3}$. Finally, an experimental prototype with unspecified thickness has an energy yield of $0.4 \mu \mathrm{J} / \mathrm{mm}^{2}$. The authors cite a pulse duration of $0.1 \pm 0.05 \mathrm{~s}$ (Krupenkin et al. 2011; Fig. 2), which, again assuming linear extrapolation, gives a power $p_{3}=4 \mathrm{~W} / \mathrm{m}^{2} \pm 2 \mathrm{~W} / \mathrm{m}^{2}$.

Depending on the requirements in situ, this helps to compare the size of the harvesters for a given output, since the excitation $\eta_{g}$ will be nearly identical as long as all three are deployed in the same environment and are harvesting from the same energy source. When the power consumption of the embedded system is known, this readily allows computation of the required minimum harvester sizes. In a practical situation, factors such as harvester cost and life time will also affect the decision making process. These 


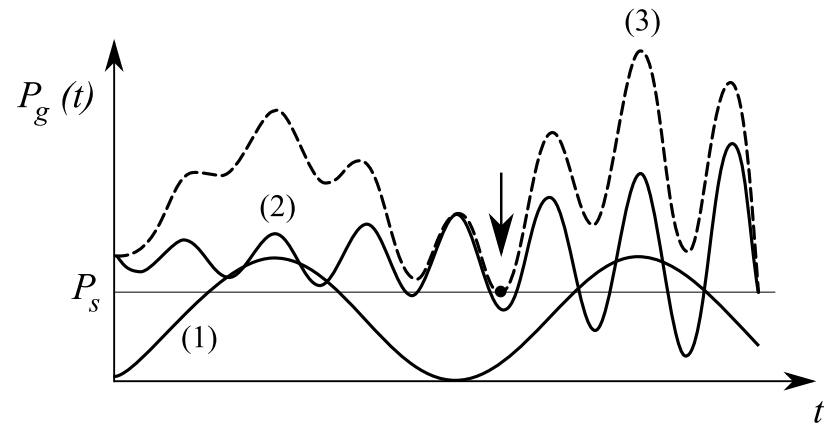

Fig. 2 A system with 2 independent generator outputs $P_{g_{1}}(1)$ and $P_{g_{2}}$ (2) profits from the harvested energy from both generators simultaneously (3), decreasing its dependency on a single generator and increasing the system's reliability

were omitted in the example for clarity on recommendation of a reviewer.

\section{Multi sourced energy harvesting}

\subsection{Single harvester drawbacks}

From a physical perspective, a large variety of different energy types are available in any environment. In an urban outdoor environment for example, various types of energy are present. Energy can be harvested from sunlight, wind, vibrations (from traffic or noise), heat from vehicle exhaust pipes etc. However, strangely enough, most ambient energy powered outdoor systems described in literature case studies are limited to a single energy source, being sunlight. Sunlight has notable advantages, such as its high energy potential, matured and widely available generators. However, it also has the obvious disadvantage of unavailability during night and cloudy moments. The classic solution applied to these problems is incorporating a battery to bridge the nights, and a solar cell with double size to charge the battery during the day aside from powering the connected system simultaneously. Often the total surface of the solar cells is over-dimensioned to compensate for uncertainties. Also the case in which the entire solar cell array is covered under a thick layer of snow for weeks in a row during winter is deliberately "forgotten" to obfuscate the apparent design flaw in this kind of single generator applications.

A second drawback of this approach is the uncertainty involved with modular energy harvesting solutions. When deploying a generator with a guaranteed nominal power, the targeted environment is often unverified for the particular energy source. Generator manufacturers provide power ratings, but seldom an exact relationship between the power output and the available ambient energy is published. This makes the integration of a generator in a new design research intensive and a project on its own. Strong efforts are necessary to translate the generator's specifications into a usable model. As a consequence, ambient energy powered systems based on a single harvester are currently only considered an option for applications which do not require high reliability.

The from an energetic point of view most viable solution for the problems above is the extraction of energy from multiple energy sources in the system's environment. When proper power conditioning circuitry is applied, the system can profit from the power generated by a multitude of generators simultaneously. For a system with a single energy harvesting generator, a stable operation is only assured when the consumed power of the system $P_{s}$ is smaller than or equal to the power generated by the generator $P_{g}(t)$ as expressed in Eq. 1 and the efficiency of the power conditioning circuitry $\eta_{p}$ :

$P_{g} \eta_{p}=p_{g} \mu_{g} \eta_{g} \eta_{p} \geq P_{S}$

When $\eta_{g}$ decreases due to a drop of the amount of ambient energy the generator is able to extract from the environment, then the system will cease to function correctly, i.e. when $\eta_{g}<\frac{P_{s}}{p_{g} \mu_{g} \eta_{p}}$.

\subsection{Transition from single to multiple harvesters}

When $n$ generators are attached to the power conditioning circuitry, this introduces $n$ (largely) independent efficiencies $\eta_{g}$ :

$\eta_{p} \sum_{k=1}^{n} p_{g_{k}} \mu_{g_{k}} \eta_{g_{k}} \geq P_{s}$

This implies the threshold at which the system fails is now dependent on the share of the dropped generator in comparison with the other generators and their respective powers:

$\eta_{g_{i}}<\frac{1}{p_{g_{i}} \mu_{g_{i}}}\left(\frac{P_{s}}{\eta_{p}}-\sum_{k \in[1, n] \backslash\{i\}} p_{g_{k}} \mu_{g_{k}} \eta_{g_{k}}\right)$

Hence, by increasing the variety of connected harvesters $n$, the stability of the system can be increased assuming $p_{\mu g_{-} 1 g_{-} 1} \eta_{g_{-} 1} \approx p_{g_{-} 2} \mu_{g_{-} 2} \eta_{g_{-} 2} \approx \ldots \approx p_{g_{-} n} \mu_{g_{-} n} \eta_{g_{-} n}$. To protect the system from the scenario that $x \leq n$ generators fail completely or experience a drop of $\eta_{g}$ with more than $90 \%$ (in the latter situation the power conditioning circuitry will be unable to convert the generator output into usable energy), the size of all generators must be increased accordingly with a factor $f$ :

$f \sum_{k=1}^{n-x} p_{g_{k}} \mu_{g_{k}} \eta_{g_{k}}=\sum_{k=1}^{n} p_{g_{k}} \mu_{g_{k}} \eta_{g_{k}}$ 
Since it was also previously assumed that all $n$ generators have an output power of a comparable magnitude, denoted by $p_{g_{-} 1} \mu_{g_{-} 1} \eta_{g_{-} 1} \approx p_{g_{-} 2} \mu_{g_{-} 2} \eta_{g_{-} 2} \approx \ldots \approx$ $p_{g_{-} n} \mu_{g_{-} n} \eta_{g_{-} n}$, the expression above can be simplified to

$f(n-x)\left(p_{g} \mu_{g} \eta_{g}\right)=(n-x)\left(p_{g} \mu_{g} \eta_{g}\right)+x\left(p_{g} \mu_{g} \eta_{g}\right)$

with $x \leq n$. Dividing the previous equation by $p_{g} \mu_{g} \eta_{g}$ and rearranging the terms gives an expression for the correction factor $f$ :

$f=\frac{n}{n-x}$

Substitution of (4) in (3) expresses how every generator in a system with $n$ generators must be over dimensioned to compensate for the failure of $x$ generators.

$\eta_{p} \frac{n}{n-x} \sum_{k=1}^{n} p_{g_{k}} \mu_{g_{k}} \eta_{g_{k}} \geq P_{S}$

In a real situation however, the previously made assumption that all generators have a comparable output power, is a rough simplification. The calculated $f$ is consequently an underestimation of the actual $f$ required for system stability.

Since

$\lim _{n \rightarrow \infty} f=\lim _{n \rightarrow \infty} \frac{n}{n-x}=1$

for a finite $x$, the reliability of the system's power supply can be increased by increasing the variety of generators $n$, assuming no strong correlation exists between energy sources. Note however that the method of decreasing the failure threshold in case of a single generator blackout consists of increasing the power of every generator by a factor $\frac{n}{n-1}$, which in practical situations corresponds to the increase of the generator's size $\mu_{g}$. And even in this situation the operation of the system is not guaranteed, since for a finite $n$, an $x+1$ failing generator will still result in the failure of the system. Since $n$ is very limited in reality as demonstrated in Sect. 8, this leaves an open problem for which a solution is suggested below.

\subsection{Influence of the system's regime}

A second point of attention is the power consumption of the system $P_{s}$. Previously $P_{s}$ has been considered constant, which is often not true for a real world electronic system. For example, a sensor node along a railway to detect the passing of trains will require more power during the day since more trains tend to pass, and thus more signals must be sent to the control room. On the other hand, during the day also more energy is available due to the presence of sunlight and the frequent passing of trains. Hence, when the size of the generators $\mu_{g_{-} i}$ needs to be calculated, it is necessary to correlate the power requirements of the system with the ambient energy available to the system. Using the worst case power consumption of the system $P_{s, \max }$ will lead to an overestimation of the required generator sizes and hence a higher cost and overall size of the system. Equation 2 shows that in case of a proportional correlation of $P_{s}$ and the efficiency of the generator $\eta_{g}$, the size of the corresponding generator may remain constant. This is unfortunately not true for most generators, which yield a high $\eta_{g}$ for short periods of time followed by comparably long periods of $\eta_{g}$ approximately equal to zero. Figure 2 demonstrates the energetic advantage of using multiple uncorrelated environmental energy harvesters.

The classic solution for both problems is the dimensioning of the generator sizes based on average power consumption. Due to the law of conservation of energy, this implies that a storage medium for energy must be introduced into the power conditioning circuitry, altering $\eta_{p}$. Since $d W=P d t$, the introduction of the time element necessary to allow averaging, shifts the equation center from power to work, changing Eq. 3 into an expression for the system's energy balance:

$\eta_{p} \sum_{k=1}^{n} \int_{T} p_{g_{k}} \mu_{g_{k}} \eta_{g_{k}} d t \geq \int_{T} P_{s} d t$

In Eq. 6, $T$ represents the period of the system. To be $100 \%$ reliable it is required that $T=\infty$, the energy storage device has an infinite capacity. It should also provide energy without having it previously stored. Obviously this is practically unfeasible as well as unnecessary since many real world electronic devices know a far shorter period grafted on environmental factors. A day or week are common periods, since these correlate strongly to natural and human behavior.

Since $T$ depends on environmental factors it cannot be calculated, however it can be fairly easily estimated based on benchmarking of the desired environment and cross referencing with common periods (day, week, etc.).

\section{Harvesting for periodic systems}

In the previous section, it was for simplicity assumed that the power consumption of the system $P_{s}$ is highly irregular. By contrast, typical electronic applications do show a regular period, either triggered by interaction or by self timed operation (e.g. clock). With the drawback of introducing uncertainty into the energy balance, Eq. 6 can be further simplified by assuming the system knows two states: an active state in which a power $P_{\text {active }}$ is consumed during a time $d$, and a standby state during which a stand by power $P_{\text {sleep }}$ is required. Examples of systems 
with these patterns include mobile phones (state during calls vs. stand by state), automated sensor modules (state during sampling of the sensor(s) and transmitting this data vs. sleep state), mp3 player (state during which music is played vs. turned off state) and many others. This does not necessarily mean that the left and right side of Eq. 6 conduct the same period. For example, a solar powered traffic counter has a harvester period of a day (the day and night cycle), while the period of the system is the average time between passing vehicles. Hence it is necessary to make a distinction between both. Let $T_{1}$ be the least common multiple period of all generators and $T_{2}$ the period of the system. Equation 6 can then be rewritten to keep the balance between produced and consumed energy:

$\eta_{p} \sum_{k=1}^{n} \int_{T_{1} T_{2}} p_{g_{k}} \mu_{g_{k}} \eta_{g_{k}} d t \geq \int_{T_{1} T_{2}} P_{s} d t$

Using the previously described elaborated regime model, the following simplification is derived:

$\eta_{p} \sum_{k=1}^{n} \int_{T_{1} T_{2}} p_{g_{k}} \mu_{g_{k}} \eta_{g_{k}} d t \geq \frac{T_{1}}{T_{2}}\left[P_{\text {active }} d+P_{\text {sleep }}(1-d)\right]$

A notable advantage of this trade off becomes apparent considering that the discrete values $P_{\text {active }}$ and $P_{\text {sleep }}$ can be found by summing the power consumptions of all system components in only 2 situations. Simulation tools such as SPICE (Simulation Program with Integrated Circuit Emphasis) (Nagel and Pederson 1973) can be used to accurately determine the power consumption of analog circuitry, while the power consumption of digital components can be found in their respective datasheets. This makes solving the left side of the equation considerably easier, assuming $T_{1}$ and $T_{2}$ are known through benchmarking. It consequently provides a powerful tool for practical system designers, as shown in Sect. 8.

\subsection{Concrete example of multi source harvesting}

Despite a theoretical approach to the problem, the presented theory can be easily applied to real world situations. Consider for example a sensor module attached to the metallic parts of the support beams of a roller coaster construction to monitor structural integrity. Logically it can be expected that all major construction elements are equipped with such sensor modules. When wired solutions are chosen, this translates to a lengthy cable installation. Exposed to the weather elements, such a setup would be prone to random errors, making a wireless solution a better choice. In such an event, the module must retrieve its power from the surrounding environment.
A quick analysis of the system uncovers three essential elements: a wireless transmitter, a corrosion sensor, and a microcontroller unit to collect measurements and transmit them wirelessly. Located in an outdoor environment, a large variety of environmental energy sources are available. In this particular situation, two sources with high potential might be identified: sunlight and track vibrations.

Sunlight, which can be harvested with a photovoltaic cell generator, provides a steady but relatively low supply of power during the day. The efficiency $\eta_{g}$ of the generator will be at maximum around noon, and nearly zero between sunset and sunrise. After benchmarking the efficiency of the solar cell $\eta_{g_{s} s}$, it may for example be found that $\eta_{g_{s}}(t) \approx e^{\sin \left(\frac{\pi}{720} t-1\right)}$ (with $t$ in minutes). The track vibrations on the other hand, harvestable using piezoelectric generators, will provide a high power spike with a very short duration. This happens when a train passes on the track segment which is monitored by the module. The efficiency of the piezoelectric harvester $\eta_{g_{-} v}$ may be determined as $\eta_{g_{v}}(t) \approx \csc ^{2}\left(\frac{t}{24 \pi}\right)$. Note that the expressions for $\eta_{g_{-} s}(t)$ and $\eta_{g_{-} v}(t)$ are rough approximations of how the parameter $\eta_{g}$ would evolve in time for a solar cell and a vibration harvester. For the sake of demonstration these behaviors were simplified to functions, whereas in actual applications $\eta_{g}$ would be measured, and thus be represented by a sequence of samples.

Neither environmental energy source is suitable to power the entire system alone. When only a photovoltaic cell would be used, its constant but low power output would prohibit high power wireless transmissions. With only a piezoelectric generator, high power wireless transmissions will be possible, but the sensor would remain unpowered during the dead time between two passing trains. Of course this problem can be solved by over dimensioning either generator and accumulating enough charge into batteries or capacitors to perform the desired action, but this would increase the cost and size of the module significantly. The presented theory can easily provide a solution here.

By combining both generators, their respective output powers can be added up to power the system as demonstrated in Eq. 7. Assume for the purpose of demonstration for the solar cell a period of 1 day $(60 \times 24 \mathrm{~min})$ and for the vibration harvester a train passing by every $4 \mathrm{~min}$. The system acquires a measurement and transmits it every 30 min, requiring a power of $2 \mathrm{~W}$ for $1 \mathrm{~s}$ and remaining in a 20 $\mathrm{mW}$ sleep state the rest of the time. Finally, let the power path circuitry have a realistic efficiency $\eta_{p}=0.8$. This is enough information to solve the problem. As explained in Sect. 5, the following expressions for the right and left side of Eq. 7 can be derived:

$$
\int_{T_{1} T_{2}} P_{s} d t=\frac{60 \cdot 24}{30}\left[2 \frac{1}{1800}+20 \cdot 10^{-3}\left(1-\frac{1}{1800}\right)\right]
$$


and

$$
\begin{aligned}
& \eta_{p} \sum_{k=1}^{n} \int_{T_{1} T_{2}} p_{g_{k}} \mu_{g_{k}} \eta_{g_{k}} d t=0.8 \\
& \quad \times\left[p_{g_{s}} \mu_{g_{s}} \int_{0}^{1440} e^{\sin \left(\frac{\pi t}{720}-1\right)} d t+p_{g_{v}} \mu_{g_{v}} \int_{0}^{1440} \csc ^{2}\left(\frac{t}{24 \pi}\right) d t\right]
\end{aligned}
$$

The generator parameters $p_{g_{s}}$ and $p_{g_{v}}$ are characteristic for the solar cell or piezoelectric generator, respectively, and may be treated as constants within the scope of this application. This leaves the generator sizes $\mu_{g_{s}}$ and $\mu_{g_{v}}$ the only variables in the equation. These variables can be found by choosing either $\mu_{g_{s}}$ or $\mu_{g_{v}}$, and calculating out the other by solving the equation. Mathematically it does not matter which parameter is chosen, but in practical situations the quantization of available generators will play an important role as explained in Sect. 8.2 (e.g. the piezoelectric generator is built up of a natural number of piezoelectric crystals). In the example above, the best solution may be found by selecting one or more piezoelectric harvesters with a combined size $\mu_{g_{v}}$ that is as close as possible to satisfying the peak power requirements of the system. The photovoltaic cell surface $\mu_{g_{s}}$ required to satisfy the rest of the power requirements can then be calculated by substituting $\mu_{g_{v}}$ in the equation and solving it for $\mu_{g_{s}}$.

\section{Balancing generator sizes}

In a system powered by complementary balanced energy harvesting, the combined power output of $n$ generators must be equal or greater than the power requirements of the system, as shown in Eq. 3. Since the parameter $p_{g}$ represents the maximum output of the generator in ideal circumstances and $\eta_{g}$ the varying environmental factors, the only factor that can influence the generator's power output is its size, $\mu_{g}$.

Considering $\mu_{g} \geq 0 ; 1 \geq \eta_{g} \geq 0$ and $p_{g}>0$, a minimum output $P_{s}$ can be generated by choosing the sizes accordingly. Assume $n$ harvesters with sizes $\mu_{1}, \mu_{2} \ldots \mu_{n}$ and provided that corresponding efficiencies $\eta_{1} \ldots \eta_{n}$ have no common zero point, e.g. $\forall t \in[0, T]: \sum_{k=1}^{n} \eta_{g_{k}}(t)>0$.

In this case, the sizes of the $n$ generators can be chosen so that at any given time at least the minimally required power $P_{s}$ is generated. A technologically optimal solution exists when the combined size of all the harvesters is minimal, which minimizes resource requirements, cost, weight and volume in the process.

The optimal size of every generator at any given time in a single period of system operation depends on the power requirement of the system, on the relative power output of the generator, and on the relative power outputs of the $n-1$ other generators feeding the system. For $n$ generators the problem can be expressed as

$\sum_{k=1}^{n} p_{g_{k}} \mu_{g_{k}} \eta_{g_{k}}(t) \geq P_{S}$

\subsection{Mathematical feasibility}

The generator's maximum outputs $p_{1}, p_{2}, \ldots, p_{n}$ and the system's power requirement $P_{s}$ can be treated as constants within this scope. This leaves $n$ variables, $\mu_{1}, \mu_{2}, \ldots, \mu_{n}$ subject to optimization. For every $t \in[0, T]$ the combined output of all harvesters $\sum_{k=1}^{n} P_{g_{k}}$ must equal or greater than $P_{s}$ so that the total harvester size $\sum_{k=1}^{n} \mu_{g_{k}}$ is minimal. Since $t$ is continuous in $[0, t]$ this presents a mathematical problem with $n+1$ variables and an infinite number of constraints, known as a semi-infinite programming problem (Hettich and Kortanek 1993). Solutions for this type of problems can be found using advanced optimization theory, but the mathematical complexity of these methods make them too cumbersome for frequent use in energy harvesting applications. However, harvesters in real world applications have discrete rather than continuous sizes (e.g. installing a solar cell of arbitrary size would be prohibited by the cost to manufacture such a custom cell). This has proven advantageous to simplify the problem. In the following sections we present a solution that is less computation intensive, that can easily be implemented in an algorithm, and is also easy to tweak to specific needs.

\subsection{Complementary balancing for 2 generators}

Because of the large number of parameters in a system with $n \in \mathbb{N}_{0}$ harvesters, we first discuss the relationships between the parameters for a system with $1(n=1)$ and 2 $(n=2)$ harvesters before presenting the generalized solution for $n$ harvesters.

For the situation where $n=1$, Eq. 9 can be simplified to $p_{g} \mu_{g} \eta_{g} \geq P_{s}$ which essentially is the simplest form of the energy balance earlier discussed, and also the simplest topology of an ambient energy powered system despite not truly being a case of complementary harvesting. If in the period $T$ the minimum efficiency of the generator $\eta_{g}$, and thus the corresponding power output $p_{g} \eta_{g}$, occurs at a $t_{m} \in[0, T]$ with $\forall t \in[0, T]: \eta_{g}\left(t_{m}\right) \leq \eta_{g}(t)$ then the size of the generator can be calculated as

$\mu_{g} \geq \frac{P_{s}}{p_{g} \eta_{g}\left(t_{m}\right)}$

on the condition that $\forall t \in[0, T]: \eta_{g}(t)>0$ and thus $\eta\left(t_{m}\right)>0$. 


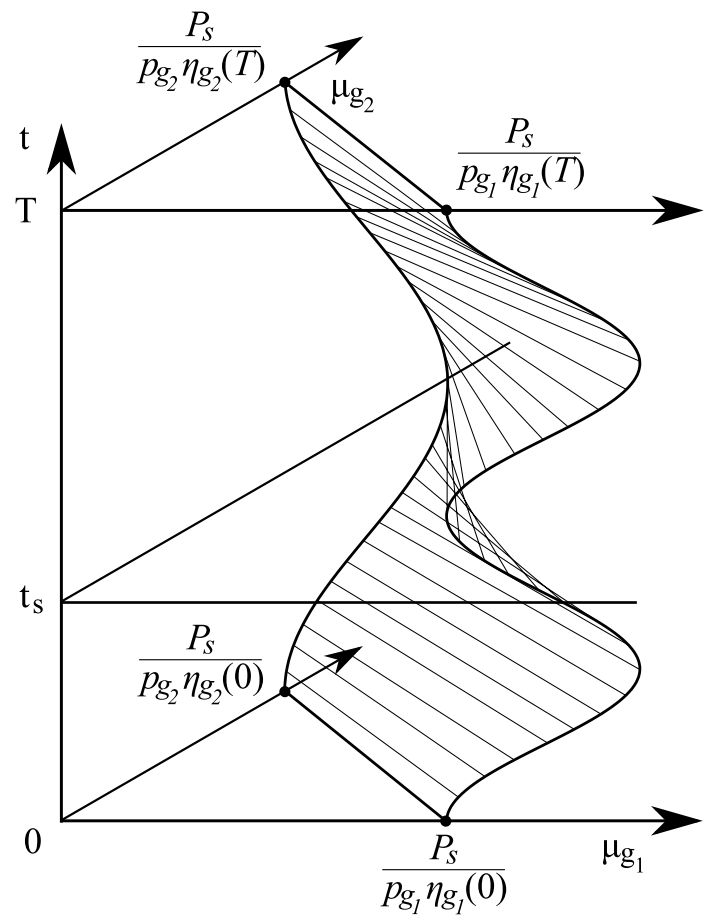

Fig. 3 Three-dimensional representation of the optimization problem as function of time $t$ and the two generator sizes $\mu_{g_{1}}$ and $\mu_{g_{2}}$. Any cross section at any given time $t_{s}$ is a line with equation $\mu_{g_{1}} p_{g_{1}} \eta_{g_{1}}\left(t_{s}\right)+\mu_{g_{2}} p_{g_{2}} \eta_{g_{2}}\left(t_{s}\right)=P_{s}$.

If $n=2$, the sum of the generated power depends on 2 generators $g_{1}$ and $g_{2}$ with each corresponding $p_{g}, \mu_{g}$ and $\eta_{g}$ parameters. Both $\eta_{g_{-} 1}$ and $\eta_{g_{-} 2}$ are functions of $t$ but statistically independent. The energy balance can be written as

$\mu_{g_{1}} p_{g_{1}} \eta_{g_{1}}(t)+\mu_{g_{2}} p_{g_{2}} \eta_{g_{2}}(t) \geq P_{s}$

again assuming that $\forall t \in[0, T]: \eta_{g_{1}}(t)+\eta_{g_{2}}(t)>0$ as condition for a solution to be found. Since the problem to solve here is finding the sizes of the generators $\mu_{g_{1}}$ and $\mu_{g_{2}}$, the equation has 3 variables $\mu_{g_{1}}, \mu_{g_{2}}$ and $t$. A graphical representation can be seen in Figs. 3, 4. For every slice in the horizontal $\mu_{g_{1}} \mu_{g_{2}}$ plane, the variable $t$ is held constant at $t=t_{s}$ and the equation of the intersection is

$\mu_{g_{1}} p_{g_{1}} \eta_{g_{1}}\left(t_{s}\right)+\mu_{g_{2}} p_{g_{2}} \eta_{g_{2}}\left(t_{s}\right) \geq P_{s}$

Transforming (11) in function of $\mu_{g_{1}}$ to retrieve the edge line equation gives

$\mu_{g_{1}}=\frac{P_{s}}{p_{g_{1}} \eta_{g_{1}}\left(t_{s}\right)}-\frac{p_{g_{2}} \eta_{g_{2}}\left(t_{s}\right)}{p_{g_{1}} \eta_{g_{1}}\left(t_{s}\right)} \mu_{g_{2}}$

which is the equation of a falling line with slope $-\frac{p_{g_{2}} \eta_{g_{2}}\left(t_{s}\right)}{p_{g_{1}} \eta_{g_{1}}\left(t_{s}\right)}$. The intersection point with the $\mu_{g_{1}}$ axis is at $\left[0, \frac{P_{s}}{p_{g_{1}} \eta_{g_{1}}\left(t_{s}\right)}\right]$ and with the $\mu_{g_{2}}$ axis at $\left[\frac{P_{s}}{p_{g_{2}} \eta_{g_{2}}\left(t_{s}\right)}, 0\right]$. This line graphically represents all combinations of $\mu_{g_{1}}$ and $\mu_{g_{2}}$ for which the

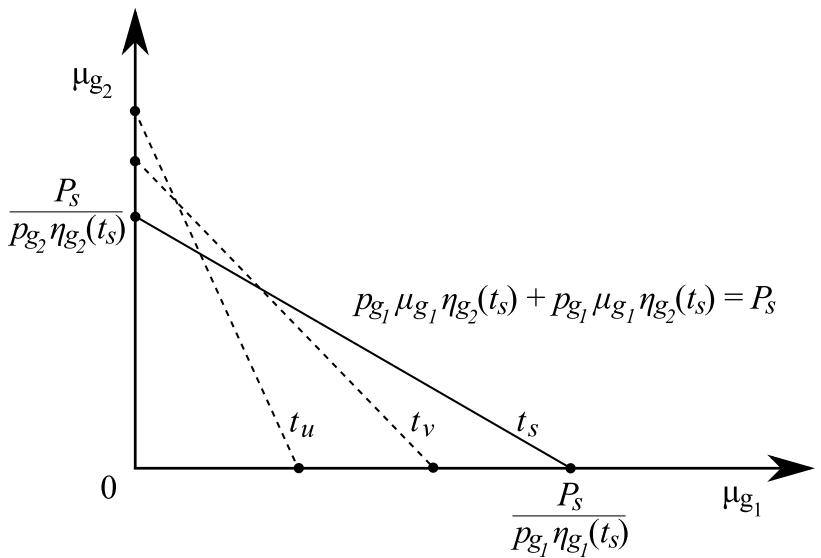

Fig. 4 Optimization problem for 3 cross sections at $t_{s}, t_{u}, t_{v} \in[0, T]$, with equation of the line given for $t_{s}$

generators $g_{1}$ and $g_{2}$ have a combined power output of $P_{s}$ at any given time $t_{s} \in[0, T]$. An optimal solution is found when both $\mu_{g_{1}}$ and $\mu_{g_{2}}$ are minimal. This point can be found by constructing a minimum curve

$m(\mu)=\mu_{g_{1}}+\mu_{g_{2}}$

and solving it for either variable. This results in 2 equations $i\left(\mu_{g_{1}}\right)$ and $j\left(\mu_{g_{2}}\right)$ :

$\left\{\begin{array}{l}i\left(\mu_{g_{1}}\right)=\mu_{g_{1}}+\frac{P_{s}}{p_{g_{2}} \eta_{g_{2}}\left(t_{s}\right)}-\frac{p_{g_{1}} \eta_{g_{1}}\left(t_{s}\right)}{p_{g_{2}} \eta_{g_{2}}\left(t_{s}\right)} \mu_{g_{1}} \\ j\left(\mu_{g_{2}}\right)=\mu_{g_{2}}+\frac{P_{s}}{p_{g_{1}} \eta_{g_{1}}\left(t_{s}\right)}-\frac{p_{g_{2}} \eta_{g_{2}}\left(t_{s}\right)}{p_{g_{1}} \eta_{g_{1}}\left(t_{s}\right)} \mu_{g_{2}}\end{array}\right.$

The equations $i\left(\mu_{g_{1}}\right)$ and $j\left(\mu_{g_{2}}\right)$ are lines in the first quadrant where a minimum positive value must exist; these minima represent the most optimal size configuration of $\mu_{g_{1}}$ and $\mu_{g_{2}}$. As example for $i\left(\mu_{g_{1}}\right)$ this yields in

$\forall \mu_{g_{1}}<\frac{P_{s}}{p_{g_{1}} \eta_{g_{1}}}: i\left(\mu_{0}\right) \leq i\left(\mu_{g_{1}}\right) \Rightarrow\left\{\begin{array}{l}\mu_{g_{1}, m}=\mu_{0} \\ \mu_{g_{2}, m}=i\left(\mu_{0}\right)\end{array}\right.$

with $\mu_{g_{1}, m}$ and $\mu_{g_{2}, m}$ the minimum sizes of $g_{1}$ and $g_{2}$ respectively. This equation proves that at any moment $t_{s}$ in the system's period, the generator with the highest efficiency is to be maximized in size, while all generators with lower efficiency will be nullified. This is expected because this generator also dominates the energy balance, and yield information for the generators is the remainder of the period is omitted, thus missing an infinite number of additional critical constraints. In the next section, we present an approximation that includes constraints from the entire period $[0, T]$.

\subsection{Balance for multiple harvesters}

Knowing the relative performance of each harvester in a system powered by $n$ harvesters is a prequirement for determination of the optimal size combination. In order to obtain representative results, the influence of each generator's size 
$\mu_{g_{i}}$ must be eliminated. For simplicity the generator size is held constant at unity $\left(\mu_{g_{i}}=1\right)$ throughout the following paragraph.

\subsubsection{Regime influence}

Assuming the $n$ harvesters powering the system are statistically independent, the optimal size of a specific harvester $g_{i}$ will be determined by 3 factors:

- the power ratio of the generator with respect to the other $n-1$ harvesters;

- the magnitude of the average power output;

- the stability of the power output over the entire period $T$.

The first and most significant factor is the ratio of a generator's power output in comparison to the combined output of the other generators at any given time. When the combined output power of all the other generators is low in comparison to a single generator, the relative importance of its contribution to the power balance of the system is high. Conversely, when a generator's output is low in comparison to the other generators, then its significance is lowered proportionally.

For a generator $g_{i}$ the power ratio $\kappa_{1}$ can be expressed as

$\kappa_{1}=\frac{1}{T} \int_{0}^{T} \frac{p_{g_{i}} \eta_{g_{i}}(t)}{\sum_{k \in[1, n] \backslash\{i\}} p_{g_{k}} \eta_{g_{k}}(t)} d t$

If $g_{i}$ has a constant output power of 0 then $\kappa_{1}=0$. Because $0 \leq \kappa_{1}<\infty$, the factor $\kappa_{1}$ is a useful first indication for the significance of a generator in the power balance of the system.

A second factor $\kappa_{2}$ is the magnitude of the average power output of the generator. Generators with high average power output have a higher contribution to the power balance of the system. Considering $\frac{1}{T} \int_{0}^{T} p_{g_{i}} \eta_{g_{i}}(t) d t$ the average power output of a generator $g_{i}, \kappa_{2}$ can be expressed as

$\kappa_{2}=\int_{0}^{T} p_{g_{i}} \eta_{g_{i}}(t) d t\left((n-1) \sum_{k \in[1, n] \backslash\{i\}} \int_{0}^{T} p_{g_{k}} \eta_{g_{k}}(t) d t\right)^{-1}$

The third factor $\kappa_{3}$ represents the degree of fluctuations of power output within a period of the system. It makes use of a continuous time standard deviation to quantize how much the generator's power output changes, as expressed in Eq. 15:

$\kappa_{3}=\left[1+\frac{1}{T} \int_{0}^{T}\left(p_{g_{i}} \eta_{g_{i}}(t)-\frac{1}{T} \int_{0}^{T} p_{g_{i}} \eta_{g_{i}}(t) d t\right)^{2} d t\right]^{-2}$
In Eq. 15 the standard deviation is easily recognizable, with $\frac{1}{T} \int_{0}^{T} p_{g_{i}} \eta_{g_{i}}(t) d t$ representing the average power output of the generator from which to calculate the deviation. The inner square ensures that only positive deviations are summed, so that positive and negative deviations do not cancel each other out. The total deviation is increased by 1 so that $\kappa_{3}$ approaches unity when the deviation with respect to the average power output approaches 0 , e.g. when the $g_{i}$ has a constant power output. Since the standard deviation is a positive value, $\kappa_{3}$ will also be a positive value with $0<\kappa_{3} \leq 1$.

\subsubsection{Harvester coefficient}

The three factors $\kappa_{1}, \kappa_{2}$ and $\kappa_{3}$ can now be combined to attribute a value to a generator $g_{i}$ as part of a system powered by $n$ generators. We define this harvester coefficient $\theta_{i}$ for any generator $g_{i}$ as

$\theta_{i} \equiv \prod_{k=1}^{3} \kappa_{k}\left[g_{i}\right]$

which corresponds to the product of the three factors $\kappa_{1}, \kappa_{2}$ and $\kappa_{3}$ we presented in the previous section. In this product, $\kappa_{1}$ and $\kappa_{2}$ will act as positive modifiers, e.g. higher values represent a better harvester performance. The factor $\kappa_{3}$ is a negative modifier causing a decrease in $\theta$ if the deviation of the generator output with respect to its average increases. Because $\kappa_{1}, \kappa_{2}$ and $\kappa_{3}$ are all positive values, also $\theta$ will be a positive value which, however, does not directly reflect the size of the corresponding generator in the system. The examples below illustrate the effectiveness and interpretation of $\theta$.

Example 1 Consider 2 generators $g_{1}$ and $g_{2}$ with respective efficiencies $\eta_{g_{1}}$ and $\eta_{g_{2}}$. To demonstrate the effect of $\theta$, the output power of both generators will be held at unity $\left(p_{g_{1}}=p_{g_{2}}=1\right)$. Assume $\eta_{g_{1}}$ a square wave with period 1 and duty cycle of $50 \%$

$\eta_{g_{1}}(t)=\left\{\begin{array}{ll}0.9 \text { if } & t<\frac{k}{2} \\ 0.1 \text { if } & t>\frac{k}{2}\end{array} \forall k \in \mathbb{N}\right.$

and

$\eta_{g_{2}}(t)=\sin \left(t+\frac{\pi}{5}\right)$

In this example the period of the system will arbitrarily be assumed 2. We can then proceed to calculating $\kappa_{1}$ for $g_{1}$ and $g_{2}$ :

$\kappa_{1}\left[g_{1}\right]=\frac{1}{2} \int_{0}^{2} \frac{\eta_{g_{1}}(t)}{\eta_{g_{2}}(t)} d t=0.602$ 


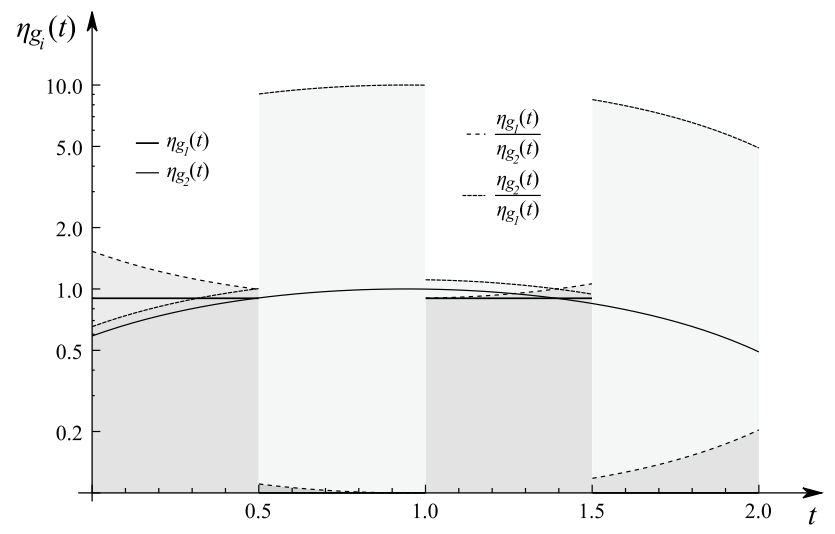

Fig. 5 Graphical representation of the optimization problem for a system powered by 2 generators with output efficiencies following a square wave and a sine arc. As shown, $0 \leq \eta_{g_{1}}, \eta_{g_{2}} \leq 1$ and $\mu_{g_{1}}, \mu_{g_{2}}=1$. Continuous curves represent $\eta_{g_{1}}$ and $\eta_{g_{2}}$ while dotted curves correspond to the power ratios. Note the high values on a log scale for $\frac{\eta_{g_{2}}}{\eta_{s}}$ as a result of division by a very small value during the low period halves of the square wave

$\kappa_{1}\left[g_{2}\right]=\frac{1}{2} \int_{0}^{2} \frac{\eta_{g_{2}}(t)}{\eta_{g_{1}}(t)} d t=4.612$

The corresponding surfaces of the ratios between $\eta_{g_{1}}$ and $\eta_{g_{2}}$ are shown in Fig. 5. Comparing the results for $\kappa_{1}$ shows a significantly higher value for $\kappa_{1}\left[g_{2}\right]=4.61$ than for $\kappa_{1}\left[g_{1}\right]=0.602$. In this example, this is attributed to the averagely higher efficiency of $g_{2}$ and the very low efficiency of $g_{1}$ during the low period halves of the square wave. Proceeding to the calculation of $\kappa_{2}$ for $g_{1}$ and $g_{2}$ results in

$\kappa_{2}\left[g_{1}\right]=\frac{\int_{0}^{2} \eta_{g_{1}}(t) d t}{\int_{0}^{2} \eta_{g_{2}}(t) d t}=0.595$

$\kappa_{2}\left[g_{2}\right]=\frac{\int_{0}^{2} \eta_{g_{2}}(t) d t}{\int_{0}^{2} \eta_{g_{1}}(t) d t}=1.650$

Again the value of $\kappa_{2}\left[g_{2}\right]$ is higher because the average power output of $g_{2}$ is higher than that of $g_{1}$. Finally, calculating $\kappa_{3}$ for $g_{1}$ and $g_{2}$ results in

$\kappa_{3}\left[g_{1}\right]=\left[1+\frac{1}{2} \int_{0}^{2}\left(\eta_{g_{1}}(t)-\frac{1}{2} \int_{0}^{2} \eta_{g_{1}} d t\right)^{2} d t\right]^{-2}=0.641$

$\kappa_{3}\left[g_{2}\right]=\left[1+\frac{1}{2} \int_{0}^{2}\left(\eta_{g_{2}}(t)-\frac{1}{2} \int_{0}^{2} \eta_{g_{2}} d t\right)^{2} d t\right]^{-2}=0.942$

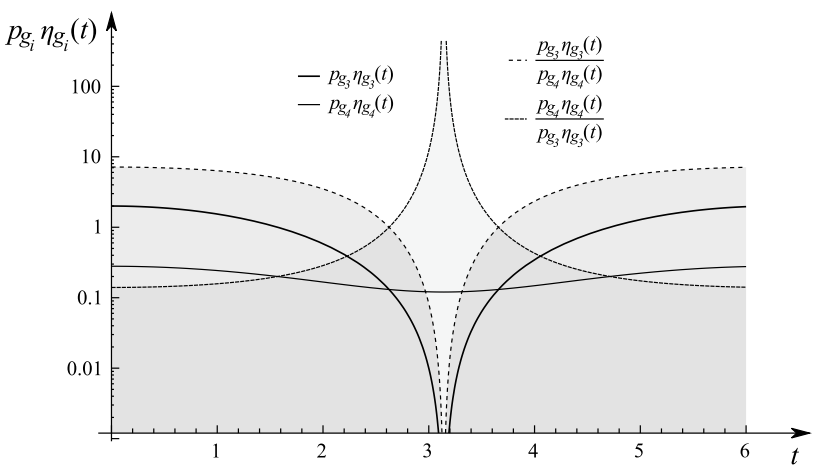

Fig. 6 Graphical optimization problem for 2 generators, one having a zero point within the interval. Note the logarithmic scale

The value of $\kappa_{3}\left[g_{2}\right]$ is lower than $\kappa_{3}\left[g_{1}\right]$ in this case because the output of $g_{2}$ is more constant than the output of $g_{1}$. Combining the values obtained for $\kappa_{1}, \kappa_{2}$ and $\kappa_{3}$ for both $g_{1}$ and $g_{2}$ results in

$\begin{aligned} \theta_{1} & =\prod_{k=1}^{3} \kappa_{k}\left[g_{1}\right]=0.23 \\ \theta_{2} & =\prod_{k=1}^{3} \kappa_{k}\left[g_{2}\right]=7.3\end{aligned}$

The values for the harvester coefficients $\theta_{1}$ and $\theta_{2}$ differ by more than a factor 30. This indicates that generator $g_{2}$ is the better choice and the preferred candidate for size optimization. Since for either generator $0<\theta_{i}$, both are able to support the system autonomously, and since neither generator has zero points in its power output, neither is critical. A counter example will be given below.

Example 2 Consider 2 generators $g_{3}$ and $g_{4}$ with power outputs $p_{g_{3}} \eta_{g_{3}}=\cos (t)+1$ and $p_{g_{4}} \eta_{g_{4}}=\frac{0.1}{2 \pi} \cos (t)$, as shown in Fig. 6. In this example, $p_{g_{-}} \geq 1$ so that $0 \leq p_{g_{3}} \eta_{g_{3}}<\infty$. Furthermore, $p_{g_{-} 3} \eta_{g_{-} 3}$ has a zero point. Calculating the factors $\kappa_{1}, \kappa_{2}$ and $\kappa_{3}$ for $g_{3}$ and $g_{4}$ in a periodic interval $[0,6]$ results in the values below.

\begin{tabular}{lllll}
\hline & $\kappa_{1}$ & $\kappa_{2}$ & $\kappa_{3}$ & $\theta$ \\
\hline$g_{3}$ & 4.186 & 4.857 & 0.311 & 6.330 \\
$g_{4}$ & 440.8 & 0.206 & 0.991 & 89.95 \\
\hline
\end{tabular}

Due to the complexity of the integral calculations, shown values are approximated using Wolfram Mathematica. It is clear that the generator coefficient $\theta_{4}$ of generator $g_{4}$ dominates the balance, indicating a strong advantage for $g_{4}$. The presence of a zero point in $p_{g_{3}} \eta_{g_{3}}$ which causes $\kappa_{1}$ 
to asymptotically reach infinity at this point, and the large spread in generator power, both contribute to this result. It is important to note here that, due to the continuous time integral for the calculation of the ratio surfaces, $\kappa_{1}$ does not result in infinity because of the asymptote to the zero point. A high value for $\theta$ may be result of a zero point for other harvesters, but may also be a result of a low average output of other harvesters.

\subsection{Computation for regime benchmarking}

While an analytical approach as presented in the previous paragraph is useful for harvesters with a very complex power output regime, in practical situations this may often not be necessary. Many real world harvesters show rather slow variations in their power output. Commonly used examples exhibiting this behavior are solar cells, Peltier elements, and so on. For a system powered by these generators it is not necessary to construct continuous functions of their power output and calculate their generator coefficients. Instead, it is more efficient to use acquired samples from benchmarking the environment of future deployment directly.

\subsection{1 $\boldsymbol{\theta}$ in discrete time domain}

The Eqs. (13), (14) and (15) can be ported to the discrete time domain by discarding the intervals between samples from the balances. Were in continuous time domain integrals have the function of calculating the surface under the curves, in discrete time domain the same can be done by summing the known samples. Because this eliminates integrals from the equations all together, the computation of $\theta$ becomes mathematically much simpler. This allows the calculation to be done on an embedded sensor node for benchmarking of the environment, for example.

If the discrete time domain measurement of the power output of a generator $g_{i}$ results in $j$ samples for $p_{g_{i}} \eta_{g_{i}}$, then the equations for $\kappa_{1}, \kappa_{2}$ and $\kappa_{3}$ can be expressed as follows:

$\kappa_{1}=\frac{1}{j} \sum_{m=1}^{j} \frac{\left[p_{g_{i}} \eta_{g_{i}}\right]_{m}}{\sum_{k \in[1, n] \backslash\{i\}}\left[p_{g_{k}} \eta_{g_{k}}\right]_{m}}$

An equal number of samples needs to be taken for every harvester for the calculation of $\kappa_{1}$.

$$
\begin{aligned}
& \kappa_{2}=\left[(n-1) \sum_{k \in[1, n] \backslash\{i\}} \sum_{m=1}^{j}\left[p_{g_{k}} \eta_{g_{k}}\right]_{m}\right]^{-1} \sum_{m=1}^{j}\left[p_{g_{i}} \eta_{g_{i}}\right]_{m} \\
& \kappa_{3}=\left[1+\frac{1}{j} \sum_{m=1}^{j}\left(\left[p_{g_{i}} \eta_{g_{i}}\right]_{m}-\frac{1}{j} \sum_{l=1}^{j}\left[p_{g_{i}} \eta_{g_{i}}\right]_{l}\right)^{2}\right]^{-2}
\end{aligned}
$$

Implementation of the equations above as algorithms in software is very straight forward since every $\kappa$ can be calculated by means of basic arithmetic functions, and the sum functions can directly be translated into software loops. The memory footprint of an embedded implementation can remain small because samples are manipulated sequentially, and no backtracking or recursion is necessary. In this regard it should be noted that in the discrete time domain, the problem of harvester size optimization is no longer a semi-infinite computation problem because the number of constraints is now finite and equal to the number of samples. However, as obtaining a solution through this method would require a vast set of equations to be solved, the method presented above is more friendly to implementation on embedded systems.

\section{Practical generator sizes}

As mentioned before, it is realistically unfeasible to integrate harvesters with arbitrary size into the system because of economic and practical limitations.

\subsection{Scale limitations}

Although some harvesters scale very well to smaller or larger sizes, such as solar cells, other harvesters will be subjected to minimal physical sizes (such as mechanical turbines). Others only scale with natural multiples of a basic harvester block, such as piezoelectric harvesters. This should be kept in mind when solving the optimization problem to avoid excessive deviations between calculated and practical total harvester sizes.

\subsection{Manufacturing limitations}

While in large volume applications it may be economically feasible to manufacture harvesters with custom sizes to precisely fit the needs of a specific electronic system, this may only rarely be the best option. Aside from the higher cost of custom harvester sizes, it is wise to overscale the harvesters to compensate for reduced generator output due to wear over prolonged periods of time. In this regard it is deemed more practical to select an off-the-shelf generator with the next higher generator output compared to the values obtained through mathematical calculation of the minimally required size.

\subsection{Combining multiple harvesters}

When designing a system, care must be taken so that all generators are placed in an optimal configuration. For example, a system deployed in a cubic enclosure and 


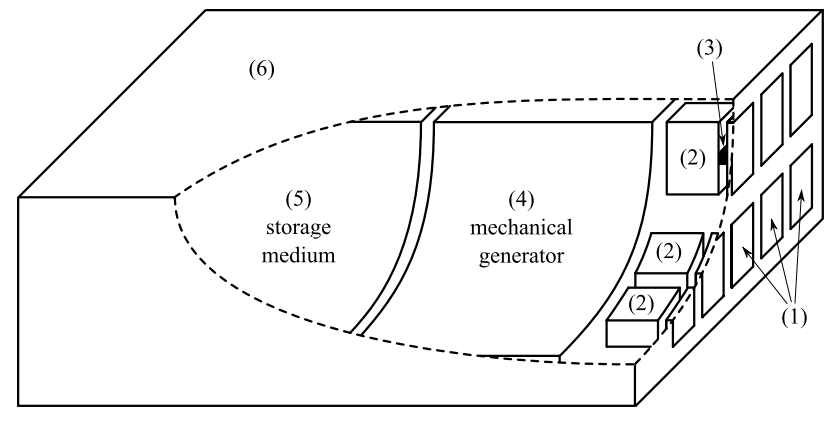

Fig. 7 Cross section of a generic ambient energy powered embedded system using a combination of different energy types and generators. The outside surface of the system is covered with photovoltaic cells (1) which are mechanically joined (3) to piezoelectric crystals to generate energy from impulses on the system's surface. The inside volume is not critical, and can be filled with magneto-mechanical generators (4). Room for an optional capacitor or battery for energy storage is also provided (5)

powered with solar energy should not be coated on all 6 sides with $\frac{1}{6}$ of the calculated solar cell surface, since for obvious reasons only 1 side will receive an optimal energy flux at any given time.

There are, however, many combinations that may turn out to be profitable from an energetic and economic perspective. A magnetic vibration harvester may for example be enclosed in a box together with the system's electronics, while the outside of the box is coated with piezoelectric or photovoltaic cells. Even photovoltaic and piezoelectric cells can be stacked since only the photovoltaic cells need to be exposed to light, as shown in Fig. 7. Listing all possible combinations would be pointless because of the application specific nature of this problem. Consequentially it should be understood that the specific requirements involved with the design of an ambient energy powered system extend far beyond limited mathematical calculations.

\subsection{Example}

To illustrate the practical usefulness of the presented theory, consider a sensor embedded systems as example. This system consists of a state of the art ultra low power microcontroller, an MSP430 from Texas Instruments (Nagy and Pederson 2003) and an SD Card to allow data storage. It is also equipped with a humidity sensor to allow monitoring of indoor humidity through the year. The sensor node is mounted to a heater, facing a window on the other side of the room. The system must be powered by environmental energy using integrated energy harvesters.

As discussed above, the unknowns in this problem are the power consumption of the system $P_{s}$, the types of harvester(s) used, their nominal power $p_{n}$, sizes $\mu_{n}$ and efficiency $\eta_{n}$. Using Eq. 8, the problem can be split in 2 halves that can be resolved separately: power consumption and power generation.

\subsubsection{Power consumption}

To sample, process and store the actual humidity value once per day, the system will remain active for only $250 \mathrm{~ms}$ per day, spending the remainder of the time dormant. From application nodes and data sheets, the active current consumption of the sensor is $5 \mathrm{~mA}$, of the MSP430 at $2 \mathrm{MHz}$ ca. $500 \mu \mathrm{A}$, and the flash SD Card $60 \mathrm{~mA}$ (Nagy 2003; Lourens et al. 2008; Foust 2004). In dormant mode (sleep with RTC enabled) the sensor and flash card are powered off and the MSP430 consumes $1 \mu \mathrm{A}$. At a system voltage of $3.0 \mathrm{~V}$ (Nagy 2003), the energy required over the entire period of one day is $308.3 \mathrm{~mJ}$ and assumed independent of the time of the year.

\subsubsection{Power generation}

Through benchmarking it is determined that the two most prominently available environmental energy sources are heat (from the heater the system is attached to) and ambient light (from natural and artificial light sources). The 3 parameters $p, \mu$ and $\eta$ must be determined for these 2 harvesters. Firstly, the parameter $\eta$ can be found either through the same benchmarking process or by consulting literature. Artificial light intensity is, with a small error, nearly constant while natural light intensity changes with the time of the year. Literature (Leslie and Martin 2009) shows an average daylight function of $H(t)=8.5 \sin (0.017 t-1.35)+12$. After scaling to match efficiency, an approximate efficiency for photovoltaic generator $g_{1}$ is obtained:

$\eta_{1}()=\frac{1}{19} \cdot 8.5 \sin (0.017 t-1.35)+12$

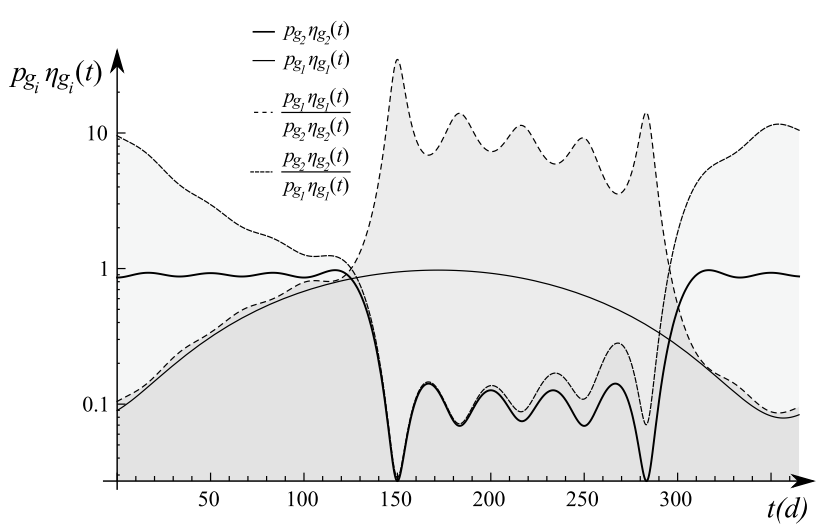

Fig. 8 Approximated evolution of harvester efficiency over a one year (365 day) period 
The efficiency of the thermoelectric generator $g_{2}$ is less complex because its efficiency is a function of the heater's activity and the room temperature. In summer it is completely turned off but acts as a heat sink, allowing reverse heat flux to flow. In winter, on the other hand, The temperature difference is high and will only see a slight drop when the room heats up. Thus, the efficiency can be expressed as a square wave signal, and approximated using a Fourier series to allow easier numeric computation. For simplicity, a 5th order approximation will be used:

$\eta_{2}(t)=\frac{5}{2}\left(1+\frac{4}{\pi} \sum_{k=1}^{5} \frac{\sin [0.003 \cdot 2 \pi(2 k-1)(t-300)]}{2 k-1}\right)+0.1$

This information can now be used to calculate the values of $\kappa_{1}, \kappa_{2}$ and $\kappa_{3}$ for both harvesters, as shown below:

\begin{tabular}{lllll}
\hline & $\kappa_{1}$ & $\kappa_{2}$ & $\kappa_{3}$ & $\theta$ \\
\hline$g_{1}$ & 2.602 & 1.005 & 0.153 & 0.400 \\
$g_{2}$ & 4.191 & 0.995 & 0.099 & 0.412 \\
\hline
\end{tabular}

Due to the complexity of the integrals, calculations were numerically approximated using Wolfram Mathematica. (Smith 1974; Krommer and Ueberhuber 1994). With only $3 \%$ difference in value between $\theta_{1}$ and $\theta_{2}$, it can be concluded that, in this specific configuration, both harvesters have a comparable efficiency and neither has a clear advantage over the other. Both values are also fairly low, indicating that both harvesters are able to power the system independently of the other if necessary. This can also be seen in graph 8, where solid lines represent the efficiencies of $g_{1}$ and $g_{2}$ as $\eta_{1}(t)$ and $\eta_{2}(t)$ for a constant $p$.

The given harvesters can be operated complementary, and balance each others maxima and minima out. This can also be found when calculating the difference of both functions, since for perfect complementation, $\frac{d}{d t}\left(\eta_{1}(t)+\eta_{2}(t)\right)=0$. The efficiency proportions are graphically represented in Fig. 8.

With the environment and the magnitude of its ambient energy sources known, the harvesters can now be dimensioned. To harvest light, a photovoltaic cel from First Solar (Von Roedern et al. 2006) is selected with a nominal power of $60 \mathrm{~W} \pm 8.3 \%$ for a cell surface of $60 \times 120 \mathrm{~cm}$. This corresponds to a power $p_{1}=83 \mathrm{~W} /$ $\mathrm{m}^{2}$. As thermoelectric harvester, an eTEG HV37 (Nextreme 2012) was selected with an extrapolated power of $222 \mathrm{~mW}$ at its maximum temperature difference of 200 $\mathrm{K}$. With a volume of $4.3 \mathrm{~mm}^{3}$, the generator power is $p_{2}=51.7 \mathrm{~kW} / \mathrm{m}^{3}$.

Based on these figures and the values of $\theta_{1}$ and $\theta_{2}$ calculated for each harvester previously, the most optimal configuration for this particular case is a (near) equal power distribution among the generators. To generate $308.3 \mathrm{~mJ}$ over its period of one day, a combined average harvester power of $5.9 \mu \mathrm{W}$ is required for power conversion efficiency of $60 \%$ (including charge-discharge efficiency of super capacitors). This can be optimally generated with $3.5 \mathrm{~cm}^{2}$ solar cells (keeping in mind day-night cycle and reduced indoor efficiency) and a single eTEG HV37 (considering $\Delta T \approx 10 \mathrm{~K}$ ).

\section{Parametrization of generator efficiency}

The efficiency $\eta_{g}$ of a generator is one of three parameters determining the total power output of a generator-the other two being the generator's normalized maximum output $p_{g}$ and its size $\mu_{g}$. To answer the question which parameters influence $\eta_{g}$, the generator must be placed in the environment from which it is supposed to extract ambient energy. Parametric dependencies can be reduced to 3 categories:

1. the amount of ambient energy the generator is able to capture;

2. the fraction of the generator exposed to the energy source;

3. variations of environmental parameters outside the regular generator scope.

During normal operation the maximum power $p_{g}$ and the generator size $\mu_{g}$ will remain constant, making $\eta_{g}$ the variable that determines the total amount of energy the generator produces since $P_{g}=p_{g} \mu_{g} \eta_{g}$. To ensure stable operation of the system, it is necessary to know the function $\eta_{g}(t)$ as precisely as possible, which is immediately the most sensitive and complex problem in the design process of an ambient energy powered system.

\subsection{Ambient energy quantity}

The amount of ambient energy in range of the generator $\phi$ is the primary factor that determines $\eta_{g}$, for a generator with a constant size $\mu_{g}$. The value of $\phi$ is independent from the generator's size, and solely a function of external factors, making $\phi$ the main reason why $\eta_{g}$ cannot be calculated by conventional means but must be experimentally determined.

In most cases $\phi$ follows patterns that can be approximated, but in some applications $\phi$ may prove to be relatively constant, for example the temperature gradient between a steam pipe in a power plant and the surrounding air. When captured with an appropriate generator such as a Peltier element, the resulting power output $P_{g}$ will be stable since $p_{g}$ and $\mu_{g}$ are also constant.

Conditions like these are exceptional however, and in any other situation the careful determination of $\eta_{g}(t)$ proves 
to be the most difficult aspect of the design of an ambient energy powered system.

\subsection{Generator exposure}

In contradiction with a common perception involving the dimensioning of generators, the exposure of the generator to the ambient energy source is subject to change. In this situation both $\mu_{g}$ and $\phi$ remain constant, but external factors reduce the amount of energy the generator is able to capture. Examples include a micro turbine getting stuck because of dust/debris in the fluid stream, or the angle of a light source changing over $\alpha$ degrees with respect to a photovoltaic cell and reducing its yield with a factor $\sin \left(\frac{\pi}{2}-\alpha\right)$.

A second case exists when the yield of the generator does not scale up linearly with $\mu_{g}$. This happens when the generator is composed of $k$ different units with each a discrete size $\mu$, where $\mu_{g} \geq k \mu$. When calculating the generator's size, this implies no arbitrary $\mu_{g}$ can be chosen as it should be a multiple of $\mu$. The discrepancy between both, $\mu_{g}-k \mu$ can de facto be considered a part of the generator that is not exposed to the energy source, with $k=\left\lfloor\frac{\mu_{g}}{\mu}\right\rfloor$. Practically, generators which exhibit this behavior, such as piezoelectric crystals for instance, will make note of $\mu$ and the maximum power that corresponds to it, allowing further calculation using the formulas above nonetheless.

\subsection{Environmental parameters}

Changing environmental parameters can be caused either by external phenomena, from which weather is the most variable, or by the system itself. Aside from very few exceptions, these system induced changes are exclusively related to warming up due to heat dissipation in hermetically sealed containers with an external energy input. Unfortunately, the system induced changes in environmental conditions are negligible compared to influences of weather, humans or natural causes. As these parameters have an unpredictable nature and are only obtainable by means of exhaustive benchmarking and field testing, it is advisable to protect the generator against external variations or make it immune to them.

As a side note, it is important to point out that those influences having a detrimental effect on the generator's performance usually also shorten the life time of the generator, effectively providing two reasons to shield them against changing environmental parameters.

\section{Future work}

Quantization of the parameters responsible for the behavior of $\eta_{g}$ over one system period $T$ is not possible with theoretical means due to the large number of factors that influence the amount of available ambient energy. The only reliable way to retrieve the required $\eta_{g}(t)$ function is benchmarking the generator in a concrete real life setting. This makes the development of an active field benchmarking platform with a test setup for the presented software algorithm an important aspect of future work in this area.

Current research focuses on the analysis of ambient energy sources together with their respective known techniques for harvesting energy from them. Determining the correlation between the normalized maximum power output $p_{g}$ and the relationship between $p_{g}$ and the amount of energy being captured by the generator is an ambiguity saturated research problem. This problem is further made complicated by the tendency of generator manufacturers to use different references for comparison. Modeling of existing energy harvesting generators and performance benchmarking where possible should allow for a set of practically usable values $p_{g}$.

Furthermore, porting the theoretical approximations in this publication to the broader perspective of generalized embedded systems allows the development of smaller, cheaper and more effective applications while bringing more electronic devices in range of energy harvesting using present day energy harvesting techniques.

Finally, the semi-infinite computation problem in the continuous time domain for complementary balanced harvesting with multiple harvesters is unresolved, despite the practical approximation presented in this paper. Additional work in theoretical mathematics may further advance this field of research.

\section{Conclusion}

This paper presented a novel model for parametrization of ambient energy harvesters. It was shown that physical and electrical properties of generators can be expressed as a maximum power and an environment dependent efficiency. The minimum size of any generator can now be calculated for a combination of system requirements and the target environment of the system using a straight forward design methodology. Successful application of the presented model in the design of an ambient energy powered electronic system demonstrated its versatility and ease of use. It was shown that the efficiency parameter can quickly be obtained by normalized benchmarking of the target environment. We concluded with the development of a practical approximation for solving the semi-infinite computation problem of balancing harvesters, and introduced the harvester coefficient as a method of quickly calculating the performance of a generator in a multi generator system. 
Open Access This article is distributed under the terms of the Creative Commons Attribution License which permits any use, distribution, and reproduction in any medium, provided the original author(s) and the source are credited.

\section{References}

Arveni (2012) Standard pulse energy harvester: AR 01, Arveni, online: http://arveni.fr/attachments/article/33/DATASHEET_Pulse_ Energy_Harvester_AR01.pdf

Arveni (2013) T. Rex: most powerful vibration energy harvester, Arveni SAS, online: http://arveni.fr/attachments/article/33/T\%20 Rex.pdf

Barosso A, Roedig UCJ (2004) Maintenance awareness in wireless sensor networks, Mobile and Internet Systems Laboratory, Univeristy College Cork

Clarke P (2010) Vibration harvesters designed to replace AA, AAA batteries, EE Times News \& Analysis, 16 July 2010

Foust F (2004) Secure digital card interface for the MSP430, Department of Electrical and Computer Engineering, Michigan State University

Fowler GA, Sherr I (2012) Tech geeks, starved for more battery power, give themselves a charge, Wall Street Journal February 2012, ISSN 009-9660, retrieved 7 March 2012

Hettich R, Kortanek KO (1993) Semi-infinite programming: theory, methods, and applications. SIAM review 35(3):380-429

Isaac, Mike (2011) Self-powering, wireless energy sensors join the internet, Wired Gadget Lab, 1 May 2011

Krommer AR, Ueberhuber CW (1994) Numerical integration on advanced computer systems, Springer, Berlin

Krupenkin T, Taylor JA (2011) Reverse electrowetting as a new apporach to high-power energy harvesting. Nature Commun 2:448

Leslie, Martin (2009) Using trig functions to model daylight hours, University of Arizona
Lourens R, Benevides J, Dillon J (2008) Using the microchip ultra low-power wake-up module, microchip application note AN879, 2008

Nagel LW (1975) SPICE2: a computer program to simulate semiconductor circuits, Memorandum No. ERL-M520, University of California, Berkeley, May 1975

Nagel LW, Pederson DO (1973) Simulation program with integrated circuit emphasis, Memorandum No. ERL-M382, University of California, Berkeley, April 1973

Nagy C (2003) Embedded systems design using the TI MSP430 series, Elsevier, ISBN 978-0750676236

Nextreme (2012) eTEG HV37 power generator datasheet, Nextreme Thermal Solutions, Inc., rev 1.0, 2012

O'Donell R (2008) Energy harvesting from human and machine motion for wireless electronic devices. Proc IEEE 96:1455-1456

Ostaffe H (2009) Practical applications of RF energy harvesting, PowerCast Corporation

Shenck NS, Paradiso JA (2001) Energy scavenging with shoemounted piezoelectrics. IEEE Micro 21(3):30-42

Smith JM (1974) Recent developments in numerical integration. J Dyn Syst Meas control 96:61-70

Tansel IN (2011) Energy harvesting from ambient vibration and ocean waves, Knovel Engineering Cases, 3 Jan 2011

Verbelen Y, Braeken A, Touhafi A (2013) Parametrization of ambient energy harvesters for complementary balanced electronic applications. In: Proceedings of SPIE 8763, smart sensors, actuators, and MEMS VI, 87631U, 2013.

Verbelen Y, Touhafi A (2013) Resource considerations for durable large scale renewable energy harvesting applications. In: Proceedings of the 2 nd international conference on renewable energy research and applications (ICRERA), 2013

Von Roedern B, Ullal HS, Zweibel K (2006) Polycrystalline thinfilm photovoltaics: from the laboratory to solar fields, National Renewable Energy Laboratory (NREL), IEEE 4th World Conference on Photovoltaic Energy Conversion (WCPEC-4), 2006 